\title{
Treatment of Schatzker Type V and VI Tibial Plateau Fractures Using a Midline Longitudinal Incision and Dual Plating
}

Kye-Youl Cho, $\mathrm{MD}^{1}$, Hyun-Sup Oh, $\mathrm{MD}^{1}$, Jae-Ho Yoo, $\mathrm{MD}^{2}$, Duk-Hyun Kim, $\mathrm{MD}^{1}$, Young-Joo Cho, $\mathrm{MD}^{1}$, and Kang-Il Kim, $\mathrm{MD}^{1}$

${ }^{1}$ Department of Orthopaedic Surgery, Center for Joint Diseases and Rheumatism, Kyung Hee University Hospital at Gangdong, Seoul; ${ }^{2}$ Seoul Sky Hospital, Seoul, Korea

Purpose: The purpose of this study was to evaluate the results of the treatment of Schatzker type V and VI tibial plateau fractures using a midline longitudinal incision and dual-plate fixation.

Materials and Methods: Ten patients with Schatzker type V and VI tibial plateau fractures treated with a midline longitudinal incision and dual plating were analyzed. The patients were followed for a minimum of one year. Clinical outcomes were evaluated using range of motion, visual analogue scale (VAS) and Knee Society Score. Radiological outcomes were evaluated using the bony union time, medial proximal tibial angle (MPTA) and posterior proximal tibial angle (PPTA).

Results: The mean VAS score was 2.2 points, and the mean Knee Society function score was 85 points at the final follow-up. The mean flexion contracture was $2.5^{\circ}$ and the mean further flexion was $125^{\circ}$. It took an average of 4 months until bony union occurred. The mean MPTA and PPTA were $90.5^{\circ}$ and $4.4^{\circ}$, respectively. There was one case of delayed wound healing, but no other complications were observed.

Conclusions: The treatment of Schatzker type V and VI tibial plateau fractures with a midline longitudinal incision and dual-plate fixation resulted in satisfactory clinical and radiological outcomes. This can be an option when treating Schatzker type V and VI tibial plateau fractures.

Keywords: Tibial plateau fracture, Schatzker type V, Schatzker type VI, Midline longitudinal incision, Dual-plate fixation

\section{Introduction}

Tibial plateau fractures are generally classified according to the method developed by Schatzker ${ }^{1}$. Schatzker type V and VI fractures are high-energy fractures often accompanied by other injuries and complications, such as postoperative inflammation, wound problems and infections. They tend to have a poor

Received October 22, 2012; Revised (1st) February 25, 2013;

(2nd) May 9, 2013; Accepted May 9, 2013

Correspondence to: Kang-Il Kim, MD

Department of Orthopaedic Surgery, Center for Joint Diseases and Rheumatism, Kyung Hee University Hospital at Gangdong, 892

Dongnam-ro, Gangdong-gu, Seoul 134-727, Korea

Tel: +82-2-440-6151, Fax: +82-2-440-6296

E-mail: drkim@khu.ac.kr

This is an Open Access article distributed under the terms of the Creative Commons Attribution Non-Commercial License (http://creativecommons.org/licenses/by-nc/3.0/) which permits unrestricted non-commercial use, distribution, and reproduction in any medium, provided the original work is properly cited. prognosis. Various surgical approaches and fixation techniques have been developed to treat Schatzker type V and VI fractures. The preferred surgical approaches have been the anterolateral single incision and anteromedial/posterolateral bilateral incisions for easier reduction of fractures and better wound healing ${ }^{2-8)}$. The midline longitudinal incision has been less frequently used because of the risk of wound problems and subsequent infections or retardation of bony union ${ }^{3,6-9)}$. However, it might be helpful for later salvage arthroplasty and could be considered in timing of fracture surgery ${ }^{2,3,5,7)}$. Several fixation methods could be used for tibial plateau fractures including unilateral fixation with a single plate, dual-plate, a hybrid external fixator or a less invasive stabilizing system (LISS) ${ }^{2,5,7,10,11)}$. All of these techniques have strengths and weaknesses and there is no clear consensus on which leads to the best outcomes.

Although poor results of the midline longitudinal incision were reported in the past, there might be some difference with the results of recent cases as surgical techniques respectful of the soft tissue care have been developed. We studied the usefulness of 
the midline longitudinal incision as an alternative approach and dual-plate fixation among patients with Schatzker type V and VI tibial plateau fractures.

\section{Materials and Methods}

\section{Patient Group}

The total number of Schatzker type V and VI tibial plateau fractures was 23 between September 2007 and June 2010. Thirteen patients received an operation with use of a single plate or screw fixation and the remaining 10 patients with use of a midline longitudinal incision on the anterior knee and dualplate fixation. We evaluated retrospectively the latter 10 patients. The average age of the patients was 51.6 years, the mean followup period was 33.7 months (range, 12 to 51 months), and there were 5 men and 5 women. The left knee joint was involved in 4 patients, and the right knee joint in 6 patients. A traffic accident was the cause of the fracture in all 10 patients. Injuries associated with the tibial plateau fractures included 1 case of femoral fracture, 2 cases of acetabular fracture, 1 case of calcaneal fracture, 1 case of open ankle fracture and 1 case of hip dislocation. Injuries associated with the knee joint included 3 cases of the medial collateral ligament injury and 3 cases of medial meniscal tear. There were 2 cases of Schatzker type $V$ fractures and 8 cases of Schatzker type VI fractures, 2 of which were open fractures. Temporary external fixation was considered in patients with multiple injuries including suspicious neurovascular injury and severe swelling accompanied by angulation and/or shortening. There were 3 cases in which patients were temporarily given an external fixator prior to the treatment with the midline longitudinal incision and dual-plate fixation. Five cases needed an additional bone graft during the operation. The mean time between injury and surgery was 9.3 days (Table 1).

\section{Surgical Operation and Rehabilitation}

Patients were positioned supine on the operating table before receiving general or spinal anesthesia. The surgical view was prepared so that the area of operation, ranging from the knee joint to the tarsal joint, was clearly visible with a tourniquet placed around the proximal thigh. We used fluoroscopy to ensure that the total length of the tibia was visible when the patient's anterior knee joint was turned towards the front side, providing about $15^{\circ}$ of rotation to the lower limb. We estimated the length of the skin incision to correspond with the length of the plate that would give full support to the region of the fracture.

A midline longitudinal incision was then performed on the distal to the knee joint. The resulting skin flap including the subcutaneous tissue and muscle fascia was lifted and the incision was extended to the anterior edge of the tibia. On the lateral side, we lifted the region ranging from the lateral joint line to the point at which the muscle of the anterior lower leg was visible. On the medial side, we lifted the region of the medial side of the tibia that was expected to contact the plate. We took great care not to damage the sartorius, the medial collateral ligament and the muscle fascia of the pes anserinus. The plateau of the tibia was exposed by lifting the meniscus after incising the meniscotibial ligament when closed reduction was needed for the articular surface. The proximal tibial metaphysis was exposed by lifting the muscle in the anterior compartment of the lower leg, and by incising the muscle fascia around the lower part of Gerdy's tubercle. In the case of subsidence of the articular surface, bone grafting was performed by creating a window in the metaphysis

Table 1. Subject Demographics

\begin{tabular}{cccccccl}
\hline Case & Sex & Age & Side & Cause of injury & Fracture type & Days from injury to surgery & Associated injury \\
\hline 1 & M & 60 & L & TA & V (open) & 13 & MCL \& MM (intrasubstance) injury \\
2 & F & 57 & R & Ped TA & V & 6 & MCL \& MM (complex) injury \\
3 & M & 39 & L & TA & VI & 6 & \\
4 & F & 41 & R & MA & VI & 6 & \\
5 & F & 46 & R & TA & VI & 9 & Fx of the femur (R) and acetabulum (L) \\
6 & F & 69 & L & Ped TA & VI & 18 & Fx of the acetabulum (R) and calcaneus (R) \\
7 & M & 58 & R & MA & VI & 7 & Hip Fx-D/L (L), open Fx of the ankle \& the calcaneus (R) \\
8 & M & 40 & L & TA & VI & 9 & MCL \& MM (radial) injury \\
9 & F & 58 & R & TA & VI & 15 & \\
10 & M & 48 & R & Ped TA & VI (open) &
\end{tabular}

R: right, L: left, TA: traffic accident, Ped: pedestrian, MA: motorcycle accident, MCL: medial collateral ligament, MM: medial meniscus, Fx: fracture, D/L: dislocation. 
and tapping the area of subsidence in the articular surface with an elevator. In the case where there was no need to make a window due to severe pulverization of the metaphysis, indirect reduction through the fracture was performed. Temporary fixation using a K-wire was conducted as required, checking the alignment of the coronal plane and the sagittal section of the tibia and fracture surface. Reduction was maintained through axial traction. Overall alignment was maintained by fixing a screw in the distal and proximal parts of the plateau and securing a locking compression plate to the medial and lateral of the tibia. In the case of severe subsidence of the fracture, rafting screw fixation was also performed to prevent further subsidence. After the plate was fixed on the medial and lateral side of the tibia, screws were inserted alternately to avoid interference between screws. We used fluoroscopy to confirm the overall alignment of the tibia and reduction of the articular surface after the fracture was fully fixed. The incision of the skin and fascia were sutured layer by layer.

Patients received immobilization with a long-leg splint for 2 weeks after the operation. The stitches were removed about 2 weeks after surgery. Range of motion exercises using a continuous passive motion machine were started 2 weeks after surgery and were increased gradually thereafter. Weight bearing was attempted at 6 to 8 weeks after surgery, and the degree of bony union was periodically observed.

\section{Evaluation Methods}

We evaluated the clinical and radiological outcomes as well as complications. Clinical outcomes were assessed with a visual analogue scale (VAS), the American Knee Society function score and range of motion of the knee joint ${ }^{12)}$. Radiological outcomes were evaluated in terms of bony union and the reduction status of the tibial plateau. Bony union was defined as the radiologically proven connections of cortical bone in more than 3 cortices during the follow-up period. Nonunion was defined as the absence of union at 6 months after surgery ${ }^{10)}$. Reduction status was determined by measuring the medial proximal tibial angle (MPTA) and the posterior proximal tibial angle (PPTA) by plain radiography $^{13)}$ (Fig. 1). PPTA was measured on the lateral view of the knee ${ }^{14)}$. Clinical complications were recorded.

The mean and range for all continuous variables were obtained with SPSS ver. 12.0 (SPSS Inc., Chicago, IL, USA). Wilcoxon signed rank test was used to evaluate the difference of MPTA and PPTA between the operated knee and contralateral knee. A $\mathrm{p}<0.05$ was considered to be statistically significant.
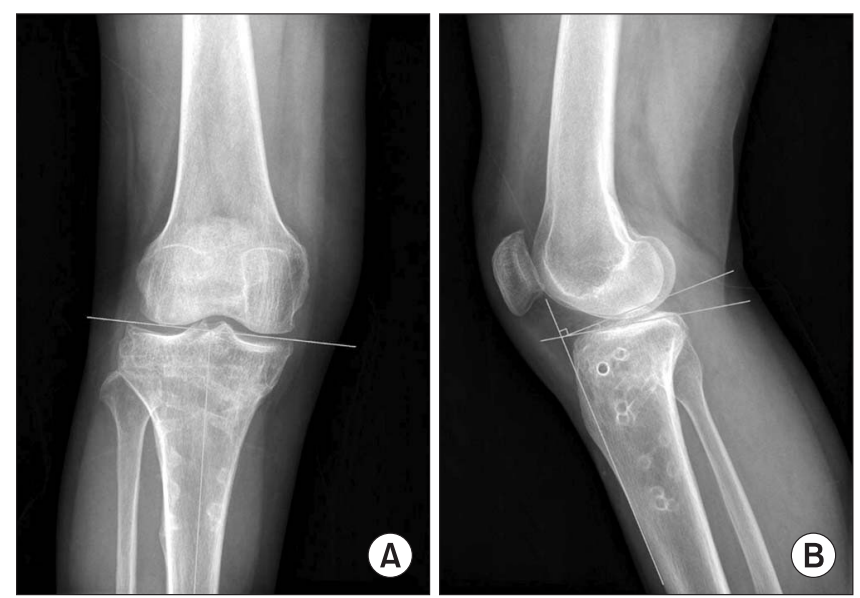

Fig. 1. (A) The medial proximal tibial angle was measured between the axis of the articular surface of tibial plateau and the anatomical axis of the proximal tibia on the anteroposterior view of the knee. (B) The proximal posterior tibial angle was measured between the articular surface of medial tibial plateau and the perpendicular line to the anterior cortical margin of the proximal tibia on the lateral view of the knee.

\section{Results}

At the final follow-up, the mean VAS score was 2.2 and the mean American Knee Society function score was 85 (Table 2). The mean range of motion of the knee joint was $122.5^{\circ}$. The mean flexion contracture was $2.5^{\circ}$ and the mean further flexion was $125^{\circ}$.

Bony union occurred approximately 4 months after surgery. The average MPTA was $90.5^{\circ}$ and the average PPTA was $4.4^{\circ}$ in the postoperative radiographs. The angles had not shown statistically significant difference between the operated knee and the contralateral uninjured knee ( $\mathrm{p}=0.075$ for MPTA, $\mathrm{p}=0.116$ for PPTA). There were no cases of reoperation due to delayed union or nonunion (Table 2).

There was one case (case no. 9 in Table 1) of delayed wound healing as a postoperative complication. This patient was carefully observed but it was determined that an additional operation was not required. Stitches were removed 4 weeks after surgery when the wound was healed. There were no cases of inflammation, infection or loss of reduction.

\section{Case Report 1}

A 40-year-old male patient was in a head-on collision and suffered a left tibial plateau fracture (Schatzker type VI) (Fig. 2A, case no. 8 in Table 1) that was accompanied by an open fracture of the right calcaneus and ankle and a left acetabular fracture with a hip dislocation. It was suspected that the patient also had 
Table 2. Clinical Outcome

\begin{tabular}{|c|c|c|c|c|c|c|c|}
\hline Case & Time to bony union (mo) & MPTA & PPTA & VAS & FC & $\mathrm{FF}$ & KS function score \\
\hline 1 & 5 & 89.2 & 10.5 & 3 & 0 & 110 & 90 \\
\hline 2 & 4 & 91.2 & 3.3 & 1 & 0 & 150 & 90 \\
\hline 3 & 3 & 87.5 & 0.3 & 2 & 10 & 120 & 100 \\
\hline 4 & 4 & 96.5 & 6.1 & 2 & 0 & 150 & 90 \\
\hline 5 & 3 & 92.5 & 10.2 & 2 & 5 & 100 & 65 \\
\hline 6 & 4 & 91.4 & 6.8 & 2 & 0 & 130 & 90 \\
\hline 7 & 6 & 91.3 & -2.3 & 3 & 0 & 125 & 75 \\
\hline 8 & 5 & 87.5 & 5.5 & 3 & 10 & 115 & 80 \\
\hline 9 & 4 & 93.5 & 2.7 & 3 & 0 & 140 & 80 \\
\hline 10 & 6 & 84.2 & 0.5 & 1 & 0 & 110 & 90 \\
\hline Average & 4 & 90.5 & 4.4 & 2.2 & 2.5 & 125 & 85 \\
\hline
\end{tabular}

MPTA: medial proximal tibial angle, PPTA: posterior proximal tibial angle, VAS: visual analogue scale, FC: flexion contracture, FF: further flexion, KS: Knee Society.

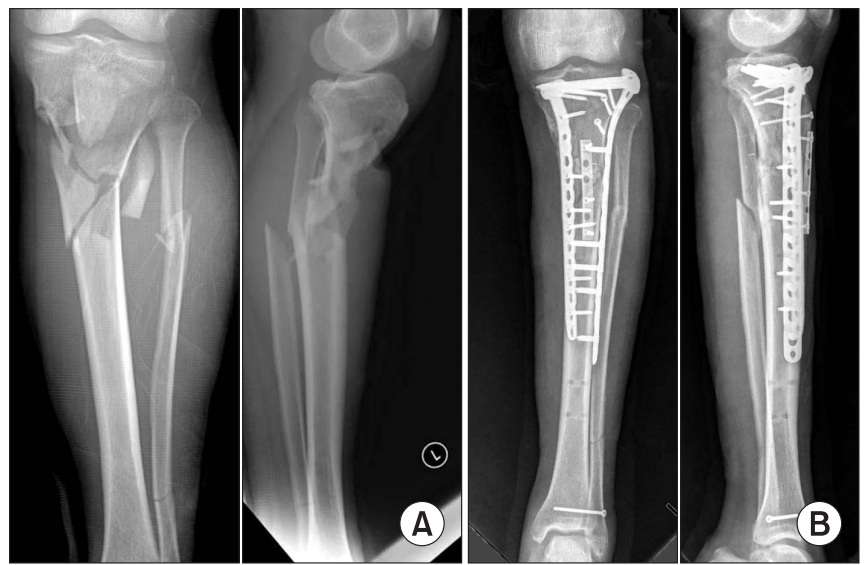

Fig. 2. Case report 1 (case no. 8 in Table 1). (A) The initial radiographs showed a severe comminuted fracture of the proximal tibia and fibula. (B) The postoperative radiographs at the seventh postoperative day showed fixation by dual plating on the medial and lateral sides.

popliteal artery rupture and nerve damage, so open exploration of neurovascular structures and temporary external fixation were performed. A midline longitudinal incision and dual-plate fixation for the tibial plateau fracture and the open reduction and internal fixation of the acetabulum were performed 7 days after the injury (Fig. 2B). Passive movement of the joints began 2 weeks after surgery. At the final follow-up, the flexion contracture was $10^{\circ}$ and further flexion was $115^{\circ}$.

\section{Case Report 2}

A 58-year-old female patient was injured in a bus accident and suffered from a right tibial plateau fracture (Schatzker type VI) (Fig. 3A, case no. 9 in Table 1). She also had injuries of the medial collateral ligament and medial meniscus underlying severe arthritic change. The injuries of the ligament and meniscus were managed with conservative care. A midline longitudinal incision and dual-plate fixation were performed 9 days after the injury (Fig. 3B, C). The removal of suture material and passive movement of the joints were postponed because healing of the wound was delayed. Passive movement of the joints began 3 weeks after surgery and the suture material was removed 4 weeks after surgery (Fig. 3D). At the final follow-up, the range of motion was $140^{\circ}$ (range, $0^{\circ}$ to $140^{\circ}$ ).

\section{Case Report 3}

A 48-year-old male patient was in a collision with a car and suffered a tibial plateau open fracture (Schatzker type VI) as well as a fracture of the fibula (Fig. 4A, case no. 10 in Table 1). Temporary external fixation was performed one day after the injury (Fig. 4B). A midline longitudinal incision and dual-plate fixation were performed 15 days after the injury (Fig. 4C). Passive movement of the joints began 4 weeks after surgery. Bony union was achieved without any other complications and the implants were removed (Fig. 4D). At the final follow-up, the range of motion was $110^{\circ}$ (range, $0^{\circ}$ to $110^{\circ}$ ).

\section{Discussion}

We studied the outcomes of the treatment for high-energy Schatzker type V and VI tibial plateau fractures using a midline longitudinal incision and dual-plate fixation. The clinical and radiological outcomes were satisfactory and there were no complications except for one case of delayed wound healing. 

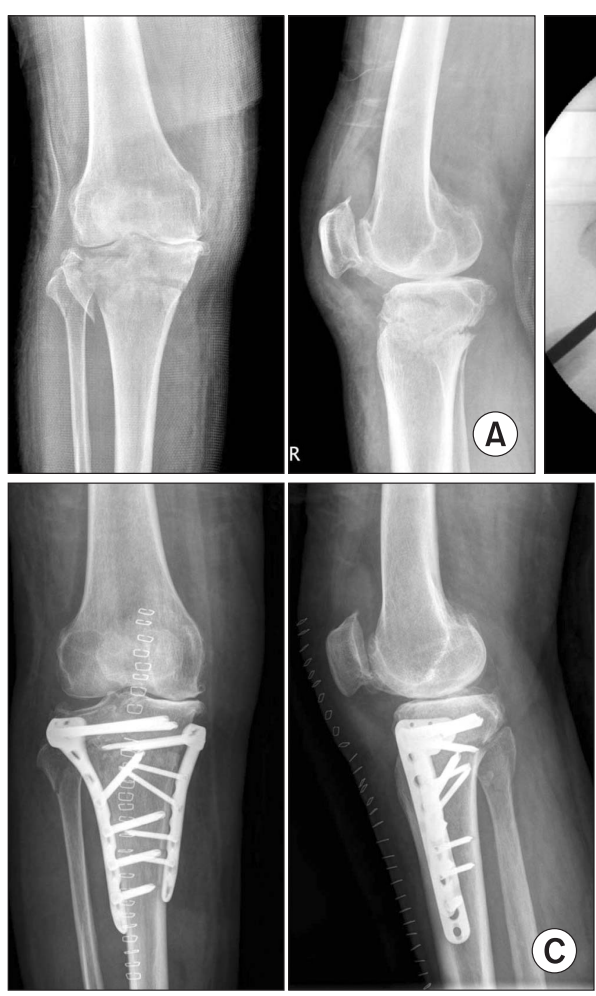

(a)
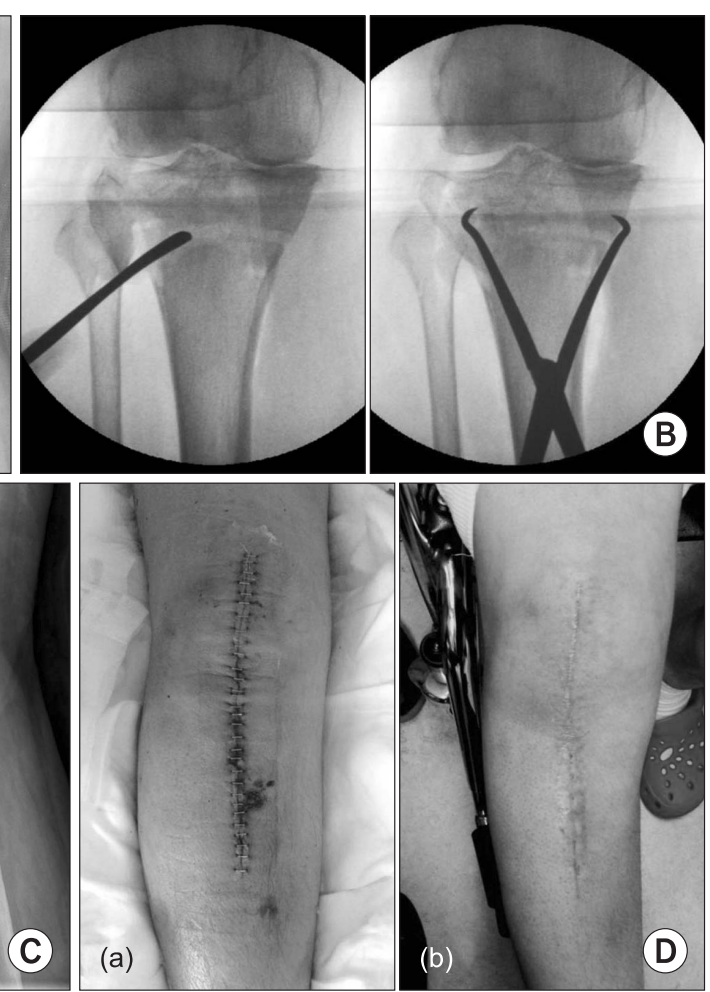

Fig. 3. Case report 2 (case no. 9 in Table 1). (A) A severe comminuted fracture of the proximal tibia was shown. (B) Intraoperative fluoroscopic images showed manipulation of the fractured fragment with a periosteal elevator. The reduced fragment was held in place with a reduction clamp. (C) The immediate postoperative radiographs showed fixation by dual plating through a midline incision. (D) (a) Delayed wound healing occurred. Marginal necrosis was observed at 7 days after surgery. (b) Complete healing of the wound was obtained without any further procedures at 4 weeks after surgery.
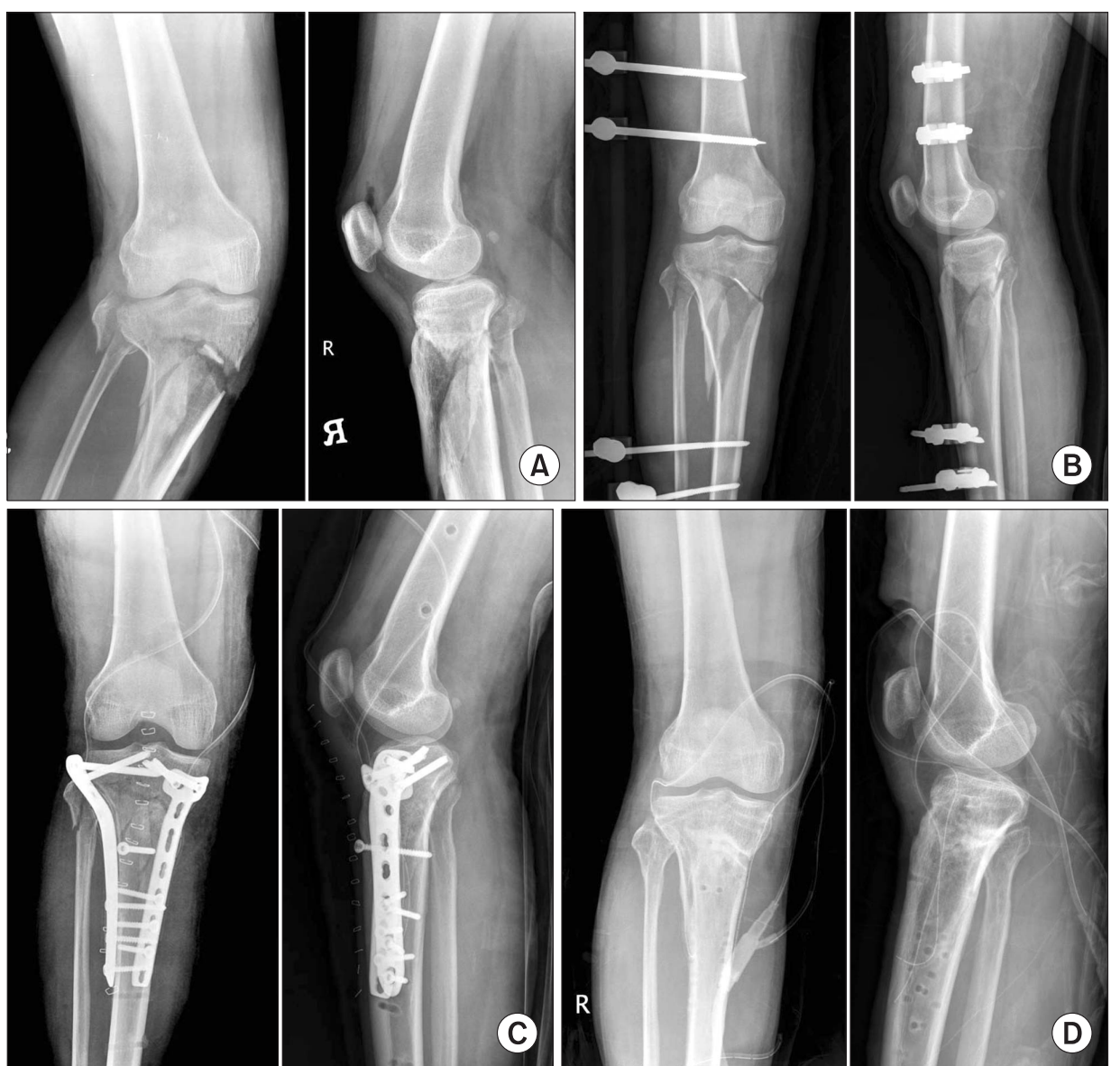

Fig. 4. Case report 3 (case no. 10 in Table 1). (A) The preoperative radiographs of the involved knee revealed a severe comminution and valgus deformity. (B) The radiographs after application of the external fixator apparatus showed the corrected alignment. (C) The immediate postoperative radiographs showed dual plating on the medial and lateral sides. (D) The final followup radiographs after removal of the internal fixation device showed sound bony union and good alignment. 
A variety of surgical approaches have been used to treat tibial plateau fractures, including the anterolateral single incision, the midline longitudinal incision and the lateral and posteromedial dual incisions. The midline longitudinal incision and the lateral and posteromedial dual incisions are mainly used for high-energy Schatzker type V and VI fractures because these fractures require reduction and fixation of both medial and lateral condyles. The midline longitudinal incision refers to the method in which the size of the incision depends on the length of the plate and the skin incision starts from the upper pole of the patella. The midline longitudinal incision has the advantage of providing full exposure of the fracture with lifting of the soft tissue flap including the subcutaneous tissue and fascia, which helps ensure accurate observation and reduction of the fracture ${ }^{3}$. However, previous studies have reported that fixation with a midline longitudinal incision can lead to infection because this approach requires a wide exposure that can damage the skin and surrounding soft tissues $^{9,15)}$. Proponents of the dual approach with lateral and posteromedial incisions have reported that this approach resulted in lower rates of inflammation and infection than the midline longitudinal incision ${ }^{6}$. The medial approach refers to the method in which the incision is performed at the rear boundary of the pes anserinus in parallel with the sartorius muscle. The anterolateral approach refers to the method in which the incision is performed about 1-2 cm lateral to the anterior boundary of the tibia; this approach has fewer wound complications because the soft tissues near the fracture site are protected. However, the lateral and posteromedial approaches also have wound complications, such as the delay in tissue healing and deep infection, though in a small number of cases ${ }^{16)}$.

A recent study suggests that the use of a hybrid external fixator to treat tibial plateau fractures leads to fewer complications of soft tissues than internal fixation ${ }^{17}$. However, an earlier study reported that the use of a hybrid external fixator also causes infection around the pin and knee joint ${ }^{18)}$. A single lateral fixation is used for Schatzker types I, II, and III fractures and it leads to satisfactory outcomes. However, maintenance of axial alignment is difficult in high-energy fractures treated with a single lateral fixation; consequently, there is the potential for malalignment, leading to a varus limb ${ }^{8)}$. Accordingly, dual-plate fixation that fixes both the medial and lateral sides is generally advocated. Horwitz et al. ${ }^{4)}$ reported good results of plate fixation of the lateral and posteromedial sides and antigliding fixation on the inside. Limbs treated with dual-plate fixation had less subsidence than limbs treated with a single lateral locking plate and dual-plate fixation had better mechanical strength compared with other fixations ${ }^{19}$. The degree of subsidence associated with the LISS method is not significantly different from that associated with dual-plate fixation ${ }^{20)}$. However, a drawback of the LISS method is that it does not perform as well as dual-plate fixation in terms of reduction of the articular surface, surgery time and soft tissue irritation by the plate ${ }^{10)}$.

Many different approaches and fixations have been used for high-energy tibial plateau fractures. Previous studies reported that dual-plate fixation with a midline longitudinal incision in selected circumstances was used but it could lead to inflammation and infection caused by the damage to the surrounding soft tissues and the large size of the incision ${ }^{2,3,5,7,9)}$. However, no patients experienced infection in our study and all patients achieved good reduction and satisfactory clinical outcomes. It can be attributable to the delayed timing of surgery with using a temporary spanning external fixator, the delicate soft tissue handling during the open reduction and internal fixation and the improved fixation hardware, such as a locking compression plate. These results could suggest that dual-plate fixation with a midline longitudinal incision can be an effective treatment for high-energy tibial plateau fractures.

Our study has some limitations. We only enrolled 10 patients and all patients were involved in some type of traffic accident. Consequently, it was not possible to determine how the mechanism of injury affected the characteristics of the resulting fractures. If our study had enrolled more patients, particularly those whose injuries were not the results of traffic accidents, our results could have been more accurate and generalizable by comparing the outcomes according to the mechanism of injury. Second, we did not enroll a control group, so we could not directly assess the relative superiority of the midline longitudinal incision and dual-plate fixation. Finally, subjects were only followed for a short period, so further studies will be needed to determine the long-term clinical and radiological outcomes.

\section{Conclusions}

The treatment of Schatzker type V and VI tibial plateau fractures with a midline longitudinal incision and dual-plate fixation of the knee joint resulted in satisfactory clinical and radiological outcomes in the current case series. This method can be considered as one of the possible treatment approaches for high-energy Schatzker type V and VI tibial plateau fractures. 


\section{Conflict of Interest}

No potential conflict of interest relevant to this article was reported.

\section{References}

1. Schatzker J. Anterior approach to the knee with osteotomy of the tibial tubercle for bicondylar tibial fractures. J Bone Joint Surg Am. 1988;70:1575-6.

2. Berkson EM, Virkus WW. High-energy tibial plateau fractures. J Am Acad Orthop Surg. 2006;14:20-31.

3. Fernandez DL. Anterior approach to the knee with osteotomy of the tibial tubercle for bicondylar tibial fractures. J Bone Joint Surg Am. 1988;70:208-19.

4. Horwitz DS, Bachus KN, Craig MA, Peters CL. A biomechanical analysis of internal fixation of complex tibial plateau fractures. J Orthop Trauma. 1999;13:545-9.

5. Marsh JL. Tibial plateau fractures. In: Bucholz RW, Heckman JD, Court-Brown CM, Tornetta P III, eds. Rockwood and Green's fractures in adults. 7th ed. Philadelphia, PA: Lippincott Williams \& Wilkins; 2009. p1780-831.

6. Stevens DG, Beharry R, McKee MD, Waddell JP, Schemitsch $\mathrm{EH}$. The long-term functional outcome of operatively treated tibial plateau fractures. J Orthop Trauma. 2001;15:312-20.

7. Tejwani NC, Hak DJ, Finkemeier CG, Wolinsky PR. Highenergy proximal tibial fractures: treatment options and decision making. Instr Course Lect. 2006;55:367-79.

8. Waddell JP, Johnston DW, Neidre A. Fractures of the tibial plateau: a review of ninety-five patients and comparison of treatment methods. J Trauma. 1981;21:376-81.

9. Young MJ, Barrack RL. Complications of internal fixation of tibial plateau fractures. Orthop Rev. 1994;23:149-54.

10. Jiang R, Luo CF, Wang MC, Yang TY, Zeng BF. A comparative study of Less Invasive Stabilization System (LISS) fixation and two-incision double plating for the treatment of bicondylar tibial plateau fractures. Knee. 2008;15:139-43.
11. Lachiewicz PF, Funcik T. Factors influencing the results of open reduction and internal fixation of tibial plateau fractures. Clin Orthop Relat Res. 1990;(259):210-5.

12. Insall JN, Dorr LD, Scott RD, Scott WN. Rationale of the Knee Society clinical rating system. Clin Orthop Relat Res. 1989;(248):13-4.

13. Honkonen SE. Indications for surgical treatment of tibial condyle fractures. Clin Orthop Relat Res. 1994;(302):199205.

14. Chung JB, Han CD, Yang IW, Che JH. Radiographic analysis of the tibial axis on the antero-posterior and lateral view of knee. J Korean Knee Soc. 2005;17:58-63.

15. Moore TM, Patzakis MJ, Harvey JP. Tibial plateau fractures: definition, demographics, treatment rationale, and longterm results of closed traction management or operative reduction. J Orthop Trauma. 1987;1:97-119.

16. Shah SN, Karunakar MA. Early wound complications after operative treatment of high energy tibial plateau fractures through two incisions. Bull NYU Hosp Jt Dis. 2007;65:1159.

17. Kumar A, Whittle AP. Treatment of complex (Schatzker Type VI) fractures of the tibial plateau with circular wire external fixation: retrospective case review. J Orthop Trauma. 2000;14:339-44.

18. Hutson JJ Jr, Zych GA. Infections in periarticular fractures of the lower extremity treated with tensioned wire hybrid fixators. J Orthop Trauma. 1998;12:214-8.

19. Ali AM, Yang L, Hashmi M, Saleh M. Bicondylar tibial plateau fractures managed with the Sheffield Hybrid Fixator: biomechanical study and operative technique. Injury. 2001; 32 Suppl 4:SD86-91.

20. Gosling T, Schandelmaier P, Marti A, Hufner T, Partenheimer A, Krettek C. Less invasive stabilization of complex tibial plateau fractures: a biomechanical evaluation of a unilateral locked screw plate and double plating. J Orthop Trauma. 2004;18:546-51. 